\title{
A unified-models analysis of the development of sexual size dimorphism in Damaraland mole-rats Fukomys damarensis
}

\author{
JACK THORLEY* AND TIM CLUTTON-BROCK
}

Author affiliations:

Department of Zoology, University of Cambridge, Downing Street, CB2 3EJ (JT, TCB)

Mammal Research Institute, University of Pretoria, Pretoria, South Africa (JT, TCB)

*Correspondent: jbt27@cam.ac.uk

Running header: Size dimorphism of Damaraland mole-rats

Abstract: Individual variation in growth rates often generates variation in fitness. However, the ability to draw meaningful inferences from growth data depends on the use of growth models that allow for direct comparisons of growth between the sexes, between populations, and between species. Unlike traditional sigmoid functions, a recently parameterized family of unified growth models provides a reliable basis for comparisons since each parameter affects a single curve characteristic and parameters are directly comparable across the unified family. Here, we use the unified-models approach to examine the development of sexual size dimorphism in Damaraland mole-rats Fukomys damarensis, where breeding males are larger than breeding females. Using skeletal measurements, we show here that the larger size of male Damaraland mole-rats arises from an increased growth rate across the entire period of development, rather than through sex differences in the duration (or timing) of growth. Malebiased skeletal size dimorphism is not unusual amongst rodents, and our measures of sex differences in size in captive mole-rats are close to sexual size differences in the wild, where size dimorphism $=1.04$. We hope our study will encourage the wide use of unified growth models by mammalogists.

Keywords: Ontogeny, sexual dimorphism, growth interval equations, cooperative breeder, non-linear mixed effects models, Bathyergidae, sociality. 
Sex differences in body size are a conspicuous feature of mammals and vary widely in

magnitude and direction between species. For example, in southern elephant seals Mirounga leonina, males can reach a body mass of 3000kg, some 5-6 times that of females (Wilson and Mittermeier 2014), whilst in other mammals, such as chipmunks and spotted hyenas Crocuta crocuta, it is the females that are the larger sex (Ralls 1976, Schulte-Hostedde 2007, Swanson et al. 2013, Kilanowski and Koprowski 2017). The developmental processes that lead the sexes to differ in size can take various forms. While, in some species, sex differences in growth are apparent during gestation and then extend throughout the rest of the lifespan (Pedersen 1980, Clutton-Brock 1991, Korsten et al. 2009), in others, they can be caused by differences in the duration of growth among adults (McNamara 1995), and in others still, they are caused by a combination of these processes (Jarman 1983, Leigh and Shea 1996, O'Mara et al. 2012).

Identifying and comparing the processes responsible for sex differences in size is now a priority for studies of sexual dimorphism and sexual selection (Badyaev 2002, Blanckenhorn 2005, Lindenfors et al. 2007, Matějů and Kratochvíl 2013), but to do this, it will be necessary to compare growth rates at particular stages of the life history of individuals. There is now a bewildering array of modelling choices to aid in this pursuit, including non-linear mixed effects models (Cole et al. 2010, Sofaer et al. 2013, Aldredge 2016) spline curves (White et al. 1999, Meyer 2005), and finite mixture models (Shotwell et al. 2016), amongst others (Tonner et al. 2017). The merits of these different approaches vary and the decision to choose one approach over another often depends on the focus of analysis, but they share a common requirement to explain growth accurately, without compromising generalizability.

To date, mammalogists have relied principally on the traditional three-parameter logistic, Gompertz and von Bertalanffy functions to characterize growth (Zullinger et al. 1984); each of which varies in the placement of the inflection point relative to the upper asymptote 
$(29.63 \%, 36.79 \%$ and $50 \%$ respectively). While it might be generally true that these threeparameter models successfully capture the shape of growth across a broad range of vertebrate taxa, it is also clear that in many cases they can return highly inaccurate parameter values. Sometimes this inaccuracy comes from the data itself: if very few individuals are sampled at the lower and upper ends of the growth curve, then three-parameter functions can struggle to recover biologically-informed size estimates at these outer ranges of the size distribution, and researchers might need to fix specific parameters when this is the case (Austin et al. 2011, Tjørve and Tjørve 2017b). In other cases, it is the model itself which is inadequate: if the true inflection point lies away from that which is forced by the logistic, Gompertz, or vonBertalanffy, or if an inflection point is absent in early life (as in monomolecular-like growth), then the three-parameter logistic models are unlikely to characterize growth accurately.

Fortunately, as the three-parameter models are part of the same sigmoid family, one can parameterize a generalized form of sigmoidal growth through the addition of a single shape parameter, ' $d$ ':

$$
S_{t}=\frac{A}{\left(1+(d-1) e^{-k\left(t-T_{i}\right)}\right)^{\frac{1}{(m-1)}}}
$$

where $S_{t}$ is the size at age $\mathrm{t}$ days, and $A, k, T_{i}$ and $d$ are the upper asymptote, the maximum relative growth rate, the age at inflection point and the shape parameter, respectively (Richards 1959). The latter shape parameter affords this so-called Richards model its flexibility by allowing the timing of inflection to vary at some point along a continuum ranging from monotonic concave to monotonic convex (Leberg et al. 1989, Gaillard et al. 1997). The threeparameter functions then sit as specific cases of this generalized Richards function (also seen as 'Chapman-Richards'). We carried out a literature search to quantify the cumulative use of the Richards function to model size-at-age data in mammals, in comparison to other two- or three-parameter growth functions. The details of this search are provided in Supplementary 
Data S1, but in brief, we looked for all studies in four mammal-focused journals (Journal of Mammalogy, Mammal Review, Mammal Study, and Marine Mammal Science) that had fitted some form of sigmoid- or sigmoid-like function to size-at-age data since 1980. As Fig. 1 shows, the flexible Richards function is only fitted in a small proportion of total studies $(20.8 \%$ as of the end of 2018), and in most cases, studies that did not fit a Richards function only fitted a single alternative function to the data $(65.8 \%)$.

However, despite its flexibility, the Richards function in its traditional form still has two major drawbacks, as outlined by Tjørve and Tjørve (2010, 2017a, see also Zach et al. 1984, Davies and $\mathrm{Ku}$, 1977). Firstly, because model parameters influence multiple curve characteristics simultaneously, they become difficult to interpret. By extension, one cannot directly compare growth parameters between Richards models fitted to different datasets, as might be desirable, for example, if one wants to compare variation in the shape and pace of growth between populations, between species, or between the sexes. That parameters influence multiple curve characteristics also introduces a second unwanted feature, namely high correlations between model parameters (Davies and Ku 1977). These drawbacks are a major obstacle in attempts to understand how and why organisms vary so widely in size and structure. In seeking to overcome the inherent limitations of the traditional sigmoid functions, Tjørve and Tjørve (2010) introduced a 'unified' Richards model whereby each parameter influences a single feature of the growth curve (Table 1). They were not the first to do so explicitly, for others had previously provided parameterizations of the Richards function where the growth rate constant was transformed into a coefficient which estimated maximum relative growth rate (Sugden et al. 1981). Even so, they were the first to develop and propose a unifiedmodels framework that incorporated the wider sigmoid family (Tjørve and Tjørve 2010, Tjørve and Tjørve 2017a). In the intervening years this unified-models approach has been increasingly adopted in the avian literature (Tjørve and Tjørve 2017b, Svagelj et al. 2019, Vrána et al. 2019), 
no doubt stimulated by the relative ease with which growth data can be collected from birds, where individuals routinely reach adult mass in the 2-3 weeks that they are bound to the nest. In stark contrast, only a single study has used the approach in a mammal (García-Muñiz et al. 2019), in spite of its obvious benefits.

Here, we use the unified-family of models to explore the development of skeletal size dimorphism in the Damaraland mole-rat, Fukomys damarensis. Damaraland mole-rats are subterranean rodents that inhabit the red arenosols of the Kalahari Desert in cooperatively breeding groups. Groups are composed of 2-41 individuals, within which a single female is responsible for all reproductive output. Paternity is often shared between 1-3 unrelated males that have immigrated into the group, the remaining individuals representing cohorts of offspring, who, having delayed dispersal, participate in burrow renovation, food acquisition and storage, group defense, and pup care (Bennett and Faulkes 2000). As in many other mammals, male Damaraland mole-rats are larger than females (Bennett and Faulkes 2000, Lindenfors et al. 2007, Young and Bennett 2013). While it has been stated that this dimorphism arises from an increased rate and a greater growth duration in males (Young and Bennett 2013), work on mole-rat growth has exclusively relied upon Gompertz and logistic equations; has often been fitted to small datasets with low temporal resolution; and has largely ignored individual variation in growth parameters (Bennett et al. 1991, Bennett and Navarro 1997, O'Riain and Jarvis 1998, Bennett and Faulkes 2000, Young and Bennett 2013, Zöttl et al. 2016). Consequently, a formal characterisation of the shape of growth in male and female Damaraland mole-rats is currently missing. The African mole-rats (family: Bathyergidae) are a particularly interesting radiation in which to examine the processes leading to sex differences in size, for patterns of growth in this family are unusually variable both within and between species (Begall and Burda 1998, Scharff et al. 1999, Bennett and Faulkes 2000, Sumbera et al. 2003). Amongst the most social species, 
including the Damaraland mole-rats and naked mole-rats Heterocephalus glaber, nonreproductive individuals can display a two-fold difference in their asymptotic mass (Bennett and Navarro 1997, O'Riain and Jarvis 1998, Zottl et al. 2016), and individuals have been shown to undergo periods of accelerated growth when reproductive opportunities present themselves (O’Riain and Jarvis 1998, Dengler-Crish and Catania 2007, Thorley et al. 2018). However, whereas Damaraland mole-rats display a male-biased size dimorphism irrespective of the female growth surge, in naked mole-rats, it is the reproductive individuals that are largest, and sexual size dimorphism (SSD) within reproductive individuals and within non-reproductive 
Morphological data were collected from captive and wild Damaraland mole-rats between October 2013 and January 2019. Our study population is located around the Kuruman River Reserve in the Northern Cape of South Africa $\left(\mathrm{S} 26.98706^{\circ} \mathrm{E} 21.81229^{\circ}\right)$, where group sizes range from $2-26$ individuals (mean $=9.47 \pm 5.44$, median $=8$ ). A captive population was founded at the reserve in February 2013 using animals sourced from the local population, and these founding individuals were either maintained in their original group or selected to create new groups, achieved through the pairing of a reproductively naïve female with an unrelated male. All individuals were part of groups housed in artificial tunnel systems made of polyvinylchloride (PVC) pipes. The pipes are modified to have transparent plastic 'windows' through which behavior can be observed, and within each tunnel system, pipes connect various compartments that serve as a nest box, a toilet, a food store and a large waste box. Depending on group size, one to three vertical pipes are incorporated into the tunnel design through which clean sand from the surrounding area can be added. Animals then replicate their natural behavior by clearing the sand from the vertical pipes and moving it through the tunnel system to the peripheral waste box, thereby gaining access to food placed behind the previously sandfilled tunnel. Animals are provisioned twice daily (ad libitum) on a diet of sweet potatoes and cucumbers. Pieces of tissue paper are also introduced into the tunnel system periodically and are readily used as nesting material. Tunnel systems are cleaned briefly every day and more thoroughly once a week.

All captive individuals used in this study were of known-age, being born into existing groups in captivity. Throughout their development, individuals were sampled for morphometrics whilst under isoflurane anesthesia. Efforts were made to repeatedly sample individuals at landmark ages, but sampling often also coincided with the collection of blood 
samples or X-rays as part of ongoing cross-sectional studies in the lab. Here, we use two morphometric measures of skeletal body size: incisor width and total body length. Incisor width, a highly repeatable measure of skull size (Young and Bennett 2010), was measured at the widest point using digital calipers $( \pm 0.1 \mathrm{~mm})$, and body length was measured dorsally from the tip of the nose to the base of the tail using a tape measure $( \pm 0.1 \mathrm{~mm})$. All morphometric samples were taken in duplicate by two observers, and we took the mean of the two measures for every sampling event. In total, the dataset on captive mole-rats comprised 3471 teeth width measures $(n=287$ females, mean/female $=6.14,1 S D=5.14$ measures; $n=269$ males, mean $/$ male $=6.35,1 \mathrm{SD}=4.57)$ and 3335 body length measures $(n=278$ females, mean/female $=6.08,1 \mathrm{SD}=4.91 ; \mathrm{n}=265$ males, mean $/$ male $=6.21,1 \mathrm{SD}=4.29)$.

Incisor width and body length data from wild mole-rats were taken as part of an ongoing capture-mark-recapture study. Groups were trapped periodically (3 to 12-month intervals) using modified Hickman traps that were baited with sweet potato and positioned into tunnel systems by digging. On capture, animals were placed into a closed, sand-filled box with other group members, and provided food and shelter, before being transported back to the laboratory where they were measured under anesthesia. Unlike the lab animals, the age of wild individuals was unknown. As such, our modelling approach relied on the parameterization of 'interval' growth equations that estimate the change in size trait across successive capture events (see below). The wild dataset comprised 447 'repeat-capture' events where incisor width was measured $(\mathrm{n}=124$ females, mean/female $=1.78,1 \mathrm{SD}=1.06 ; \mathrm{n}=128$ males, mean $/ \mathrm{male}=$ $1.77,1 \mathrm{SD}=1.04$, and 448 similar measures for body length $(\mathrm{n}=287$ females, mean $/$ female $=$ $1.78,1 \mathrm{SD}=1.06 ; \mathrm{n}=269$ males, mean $/$ male $=1.76,1 \mathrm{SD}=1.04)$

Note that for females, we only include morphometrics taken when individuals were reproductively naïve (i.e. not a dominant, reproductively active female), as skeletal growth curves of reproductive females are known to change around the acquisition of a breeding 
position (Young and Bennett 2010, Thorley et al. 2018). Reproductive females can be readily identified by their perforate vagina and prominent teats. As similar status-related changes in skeletal traits have not been reported in males, a similar exclusion is not made for males.

\section{Growth in captivity: the unified sigmoid family}

We fitted five forms of unified growth function to incisor width and body length data: Ulogistic, U-Gompertz, U-von Bertalanffy, U4, and U-Richards (Table1, see Tjørve and Tjørve, 2017a for full details). The U4 is likely unfamiliar to most readers, but simply represents a three-parameter model where the inflection point falls to $63 \%$ of the upper asymptote, an arbitrary position not covered by the other three-parameter models. This serves to illustrate that one can parameterize any three-parameter model from the U-Richards model according to the proportion of the asymptote at which the inflection points falls $\left(S_{i}=d^{1 /(1-d)}\right) ; S_{i}=0.63$ when $d=$ 4. One might wish to do so with small datasets, where the fitting of the four-parameter URichards might struggle to converge. Each of these forms of unified growth represents the socalled $\mathrm{T}_{\mathrm{i}}$-form, with $\mathrm{T}_{\mathrm{i}}$ estimating the timing of inflection. The functions can equally well be presented in a $\mathrm{W}_{0}$-form, which would estimate the weight at birth. These forms differ only in the specification of their location parameters, $T_{i}$ and $W_{0}$, which shift the growth curve horizontally. One needn't fit both equations, as one form can easily be estimated from the other (Appendix A in Tjørve and Tjørve, 2017a).

Each unified growth function was specified as a non-linear mixed effect model (NLMM). Separate models were fitted to the male dataset and female dataset for each skeletal trait, and for each unified function, random effects of $A, k$ and $T_{i}$ were first specified at the level of the individual, so that variation in growth parameters between individuals was estimated. Random effects were assumed to be independent of one another. As other studies have found 
that some growth parameters vary little between individuals (Sofaer et al. 2013), we also refitted each function with different combinations of random effects (e.g. only random effects at the level of the individual for $A$ and $k$ ), judging the best fitting model by AIC criteria and likelihood ratio tests; this model was taken to represent the best unified function for a given trait in each case. The best-fitting of the five functions were likewise compared by AIC (Burnham and Anderson 2002).

To minimize heteroscedasticity a power variance function was also consistently applied as per English et al. (2012), and significantly improved the fit of models. Specifically, nlme's varPower function was set so that observations were assumed to vary normally about a mean to $\Upsilon$ and $\rho$ :

Because of the nature of growth data, we also identified strong temporal autocorrelation models the residual at age $t\left(\varepsilon_{t}\right)$ as a function of the residual at age $t-1\left(\varphi \varepsilon_{t-1}\right)$, along with a noise term $\left(\eta_{\mathrm{t}}\right)$ as:

$$
S_{t} \sim \mathrm{N}\left(\mu=\mathrm{E}\left(S_{t}\right), \sigma=\Upsilon \cdot \mu^{\rho}\right)
$$

within individuals. We therefore also included an AR-1 auto-correlation structure, which

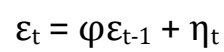

Here, $\varphi$ represents the correlation between residuals one unit apart in time and must be estimated from the data. In our study, $\varphi$ varied from $0.39-0.54$ to when considering the bestfitting models of each trait.

In addition to the unified-functions, we also fitted a general additive mixed model (GAMM) with a smoother term for age to each of the morphological datasets. As GAMMs allow for a flexible trajectory of size with age, we compared the fit of the GAMMs to the NLMMs to check whether certain features of the raw data were being poorly estimated by the unified models. In each GAMM, we specified individual identity as a random effect, and the 
age smoother was set with 6 knots.

\section{Growth in the wild}

To investigate the extent to which our results in captivity could be considered representative of growth under natural conditions, we then modelled growth of wild mole-rats. However, mole-rats captured in the wild were of unknown age, and we had no information that could be used to infer age with any confidence (e.g. tooth wear, Hart et al. 2007). This being the case, we could not use conventional age-dependent models of growth for wild mole-rats, and instead chose to use the 'interval equations' set out by Schoener and Schoener (1978, see also Fabens 1965). These interval equations have as their dependent variable the size of the animal at the end of a time interval (here the recapture of previously caught mole-rat), with the size at the beginning of the interval, and the length of the interval, forming the two independent variables. Each such model then estimates two growth parameters, the population-level asymptotic mass and the population-level growth rate constant (excepting any random terms that might be included in a mixed-effects framework).

We fitted two forms of interval equation to skeletal data from wild mole-rats, a von Bertalanffy parameterization and a logistic parameterization (Schoener and Schoener, 1978). For the von Bertalanffy, the size of an individual on recapture was modelled as:

$$
S_{2}=A-\left(A-S_{1}\right) e^{-k \cdot D},
$$

and for the logistic as:

$$
S_{2}=A \cdot S_{1} /\left(S_{1}+\left(A-S_{1}\right) \cdot e^{-k \cdot D}\right),
$$

where $S_{1}$ and $S_{2}$ are size at capture 1 and 2 (recapture) respectively, D is the time interval between capture events, and $A$ and $k$ are the growth parameters to be estimated. As above, models for males and females were fitted separately for both incisor width and body length, and AIC comparisons were used to determine relative fit. In all cases, models also specified random terms at the level of the individual for both $A$ and $k$. Also note that $k$ here is not directly 
comparable to $k$ in the unified-growth models employed for captive mole-rats; it is nonetheless instructive for making comparisons between the sexes.

All NLMMs were fitted in the nlme package in R v. 3.5.2 (R Core Team, 2018). GAMMs were fitted in $m g c v$. We provide example code for the fitting of NLMMs in the Supplementary Data. We provide the mean \pm 1 SEM for all estimates unless otherwise stated. The research carried out in this study was approved by the University of Pretoria animal ethics committee (permit numbers EC089-12 and SOP-004-13) and align with ASM guidelines (Sikes 2016). The data used in the manuscript is deposited in the University of Cambridge repository, doi: 10.17863/CAM.37910.

\section{Results}

The various forms of unified-model differed widely in their ability to capture variation in mole-rat skeletal growth in captivity (Table 2). As expected, there was a general tendency for the unified-Richards function to outperform the three-parameter functions, as for male incisor width, female body length, and male body length; the exception being shown by female incisor width, which was best explained by a U-von Bertalanffy function. When directly compared, the best-fitting models for males and females indicated that the greater asymptotic mass of males in captivity (Table 3) arose principally from an increased growth of males across development (Table 3, $g_{\max }$; Fig. 2 a, b). In contrast, there was no clear trend for males to prolong the duration of their growth beyond that of females. For incisor width, females were predicted to reach $90 \%$ of their asymptotic mass by 1.44 years of age, as compared to 1.50 years for males, whereas for body length, $90 \%$ asymptotic mass was reached at 1.13 and 1.09 years of age for females and males, respectively. Fitting age-related smoothers to morphological data using GAMMs confirmed that the growth rates of males and females had converged by the time individuals were approximately one and a half years of age (Fig. 2c). 
Estimates of growth parameters showed substantial variation among models according to sex and skeletal trait (Table 3, Supplementary Data S2 and S3). Variation was particularly pronounced for the inflection point, $T_{i}$, with a standard deviation among models consistently above 65 days (female incisor width, $1 \mathrm{SD}=77.11$; male incisor width, $1 \mathrm{SD}=74.35$; female body length, $1 \mathrm{SD}=82.08$; male body length, $1 \mathrm{SD}=67.28$; we could not use the coefficient of variation as some inflection points were negative). For incisor width, the best-fitting models suggested that for females the inflection point occurred at 4 days of age, by which point females were $29.6 \%$ of their estimated upper asymptotic size $(d=0.6667)$, whereas for males for males, inflection occurred at 112 days of age, $43.8 \%$ of their asymptotic size $(d=1.45)$. Marked contrasts in $T_{i}$ and $d$ were also shown in the best-fitting models for body length (Table 3 ). One might expect these differences in parameter estimates to translate into sex-specific differences in the shape of growth, but with the very low relative growth rates in mole-rats $(k)$, which reduces the magnitude of changes either side of the inflection point, this is not the case (Fig. 2). On the contrary, aside from the higher absolute growth rate of males, the plotting of predicted curves highlighted that male and female size trajectories are extremely similar, and any inflection values estimated by growth models only generated minor deviations in growth rate. These patterns were corroborated by GAMMs, which indicated that in mole-rats postnatal growth rate is fastest at birth and declines thereafter: mole-rat skeletal growth does not display a strong inflection. are estimated at the point of inflection- should be interpreted with caution. We can gain some insights from the estimates of $k$ in the incisor width models, as the inflection point is positive in all models, and thus $k$ is estimated within the range of the data. Doing so, we see that the relative growth rate of males is lower than females, but only modestly so. In contrast, body length models frequently returned negative inflection values, and it is therefore not intuitive to 
use estimates of $k$. Consequently, we chose to use GAMMs and model predictions to make inferences about sex differences in the pace of growth, which as noted above, are minor. For other species with a stronger inflection in growth during development, $k$ will provide a more useful metric.

Unlike the other parameters, variation among models in the upper asymptote $(A)$ was small. This was reflected by low coefficients of variation across models: CV was always less than 2.02 in A (female incisor width, $\mathrm{CV}=0.98$; male incisor width, $\mathrm{CV}=2.02$; female body length, $\mathrm{CV}=0.41$; male body length, $\mathrm{CV}=0.43$ ). Using the population-level estimates for $A$, we estimated a male-biased skeletal size dimorphism of 1.14 for incisor width, and 1.06 for body length. There was also considerable between individual variation in $A$, which was always retained as a random effect, as was $k$ (Supplementary Data S4, S5).

The modelling of growth in the wild supported our interpretations of mole-rat growth in captivity (Supplementary Data S6; Fig. 3 and Fig. 4). Firstly, we found that the von-Bertalanffy interval equations provided a better fit to the data than the logistic curve, in keeping with the male $k=0.0044 \pm 0.0002$ ), and these differences contributed to a male-biased body length dimorphism of 1.04 (female $A=18.83 \pm 0.15$; male $A=19.60 \pm 0.10$ ). Differences in growth rate were not detected for incisor width (female $k=0.0040 \pm 0.0002$; male $k=0.0039 \pm$ 0.0002), but asymptotic body length was similarly male biased (female $A=6.05 \pm 0.06$; male $A=6.79 \pm 0.06$ ), equating to an SSD in body length of 1.12. As a result, mole-rats in the wild are skeletally similar in size to the mole-rats in captivity, and SSD is invariant with respect to location.

If we compare SSD in body length of mole-rats (in the wild) to other rodents using 358 information compiled by Schulte-Hostedde (2007), we see that mole-rats lie at the $75^{\text {th }}$ 
percentile of the distribution (Fig. 5), where 1 represents a 1:1 male to female ratio. The mean sexual size dimorphism in rodents is $1.011 \pm 0.004$, though some families contribute more heavily to this distribution than others.

\section{Discussion}

Our study provides an in-depth examination of sex differences in Damaraland mole-rat growth. Focusing on skeletal traits, we show that the greater size of males of this species is caused principally by their higher absolute growth rate and that contrasts in the duration of growth between males and females were minimal (as for incisor width), or absent (as for body length). Our modelling approach relied upon a unified framework of sigmoid equations that has seldom been applied to mammals despite several attractive features: unified parameters are directly comparable across members of the family; each parameter affects a single curve characteristic; and correlations between parameters are reduced. By incorporating these unified equations into non-linear mixed effects models, we could also quantify the considerable individual variation in growth that has documented in African mole-rats (Jarvis et al. 1991, O'Riain and Jarvis 1998, Bennett and Faulkes 2000, Zottl et al. 2016). Although our use of these equations was restricted to known-age individuals sampled in captivity under ad libitum feeding conditions, a broader examination of mole-rat growth in the wild suggested that patterns of growth in captivity were largely consistent with those operating in natural populations.

While previous studies have assumed Damaraland mole-rat growth to follow a logistic or Gompertz trajectory (Bennett and Navarro 1997, Young and Bennett 2013, Zöttl et al. 2016), we show that for skeletal traits, mole-rat growth is more monomolecular-like in form (see also Thorley 2019 for an examination of body mass), lacking a strong inflection point across 
development. Strictly speaking, most of the unified equations did identify an inflection point in early life, but when plotted, it became apparent that any such inflections were modest. Likewise, an age-related smoother fitted to the raw data did not yield an inflection point, implying that postnatal growth of mole-rats is fastest at or around birth and declines thereafter. It is not unlikely that many other mammal populations display a similar monotonic concave growth trajectory, but with only a handful of studies formally comparing multiple growth functions on any given taxa in the published literature, it is difficult to know the extent to which this is the case. There are examples we can draw upon, nonetheless. Meerkats Suricata suricatta, roe deer Capreolus capreolus, and spotted hyenas have all been shown to display monomolecular growth in body mass and skeletal traits (Duncan et al. 2000, English et al. 2012, Swanson et al. 2013), and Gaillard et al. (1997) documented various other examples in a comparative analysis. Interestingly, in our own study, the U-logistic or the U-Gompertz curves did not unduly affect estimates of asymptotic mass, but this is certainly not true for other mammalian datasets where it can sometimes deviate substantially from the empirically overreliance on the traditional three-parameter functions is unwise when a flexible alternative is readily apparent in the form of the Richards function. Previously, a caveat of the Richards functions was the interdependence of estimated parameters, which rendered the interpretation of growth patterns challenging. Parameterized in its unified form, this no longer holds, and as a result we suggest that the U-Richards models should form the default sigmoid-like growth curve for mammals where no a priori knowledge of the shape of growth is available, as has already been proposed for birds (Tjørve and Tjørve 2010, Tjørve and Tjørve 2017). growth parameters of male and female mole-rats. One of our initial intentions in doing so was 
to directly compare sex differences in $k$, which represents the relative maximum growth at the time of inflection. Our analysis of incisor width found a slightly higher $k$ in females, but in our analysis of female body length the inflection point was negative and therefore represents some arbitrary timepoint before parturition. In this context, knowledge of $k$ carries no relevant information. This presents a caveat in using the unified approach when growth it not clearly sigmoidal, and where this the case we recommend that researchers instead make use of model predictions and additional growth estimates that can be readily extracted from the model output, such as the time to $90 \%$ asymptotic mass (see also France et al. 1996).

In rodents, as in mammals generally, it is typically males that are the larger sex (Lindenfors et al. 2007, Schulte-Hostedde 2007). In some cases, the SSD of adults is produced primarily by contrasts in the duration of growth, as for many terrestrial herbivores (McNamara 1995), but in most mammalian species, it seems that both a prolonged growth duration and an increased growth rate contribute to SSD (Badyaev 2002). Our results suggest that Damaraland mole-rats are somewhere intermediate, because although we clearly demonstrate the increased growth rate of males, the evidence for a prolonged growth duration is weak, and if apparent, its influence on sexual size dimorphism is minimal.

Although the magnitude of male-biased size dimorphism in Damaraland mole-rats (m:f SSD of 1.04 for body length in wild animals) is not unusual amongst rodents, it is unexpected in a cooperative breeder where reproductive skew is greater in females than males (Bennett and Faulkes 2000). Conventional sexual selection theory would therefore predict that females should be larger than males, with associated masculinized traits (Hauber and Lacey 2005, Clutton-Brock et al. 2006). However, just as in mole-rats, other cooperatively breeding vertebrates with greater female reproductive skew also fail to display reversed size dimorphism (Young and Bennett 2013), and so seems that unlike in polygynous societies where males are large and heavily armed, skew cannot account for sexual size dimorphism in cooperative 
breeders. One possible explanation for the absence of any association between skew and size in cooperative breeders is that females are trading off investment in growth against investment in reproduction (or future reproduction, i.e. fecundity selection versus sexual selection), but empirical tests of this assertion are currently absent. Comparisons with growth in the wider bathyergid family could prove particularly useful in helping to understand the ecological pressures shaping size dimorphism in Damaraland mole-rats, for with such large variation in SSD spread across species differing in their mating systems (Begall and Burda 1998, Scharff et al. 1999, Bennett and Faulkes 2000, Sumbera et al. 2003), it seems plausible that SSD reflects selective pressures operating in contemporary populations, rather than an evolutionary relic of selection in evolutionary time- or as Blankenhorn (2005) puts it, a 'ghost of SSD evolution past' (i.e. the phylogenetic signal in SSD is weak).

of its estimation has been a focus of interest for decades (Parks 1982, Starck and Ricklefs 1998). Studies of domesticated mammals have been particularly influential in driving this body of work, but surprisingly few studies of mammals have gone beyond the characterization of growth to ask ecologically informed questions. In one of the few exceptions, Gaillard et al. (1997) used a formulation of the Richards model to investigate the relationship between precocity and the form of growth across 69 mammal species. Their results suggested that peak relative growth rate is relatively earlier in precocial than altricial species, but this pattern only held at the higher taxonomic level of Order. But other examples are conspicuously rare. We suggest that in the era of open data, the unified family of equations that we present in this study can serve as a powerful tool to better investigate mammalian growth in a comparative setting, and we encourage its widespread adoption by mammalogists. 
We are grateful to the Kalahari Research Trust and the Kalahari Meerkat Project for access to

facilities in the Kuruman River Reserve, South Africa, and to Marta Manser, who helps to maintain the field site with grants from the University of Zurich and the Swiss National Science

Foundation. During the period of this study project, the research on Damaraland mole-rats has been supported by a European Research Council Advanced Grant (Nos 294494 and 742808)

to Tim Clutton-Brock. Jack Thorley was funded by a Natural Environment Research Council (UK) Doctoral Training program during the inception of the work. We would like to thank the

Northern Cape Department of Environment and Nature Conservation for permission to conduct the research (FAUNA 1020/2016), and Philippe Vullioud, Markus Zöttl, Dave Gaynor and Tim

Vink, and numerous other research assistants, for their historic and continued work with the mole-rats in the Kalahari.

\section{Supplementary Data}

Supplementary Data S1. Methods for the literature search of the use of different sigmoid growth formulations in the mammal literature.

Supplementary Data S2. Instantaneous growth rate equations for unified models of sigmoid growth.

Supplementary Data S3. Instantaneous growth rates (growth velocity) for the Unified growth models applied to female incisor width, male incisor width, female body length, and male 480 body length.

Supplementary Data S4. The best fitting random effects structure for each form of Unified growth model fitted to skeletal traits.

Supplementary Data S5. Estimates for random effects, temporal autocorrelation, and power of variance covariate in models of skeletal growth on captive Damaraland mole-rats.

Supplementary Data S6. Parameter estimates and model comparison for 'interval equation' models of growth in wild Damaraland mole-rats. 
Aldredge, R. A. 2016. Using non-linear mixed effects models to identify patterns of chick growth in House Sparrows Passer domesticus. Ibis 158: 16-27.

Austin S. H., Robinson, T. R., Robinson, W. D. and R. E. Ricklefs. 2011. Potential biases in estimating the rate parameter of sigmoid growth functions. Methods in Ecology and Evolution 2: $43-$ 51. doi: 10.1111/j.2041-210X.2010.00055.x.

Badyaev, A. V. 2002. Growing apart: an ontogenetic perspective on the evolution of sexual size dimorphism. Trends in Ecology \& Evolution 17(8): 369-378. doi: 10.1016/S0169-5347(02)02569-7.

Begall, S. and H. Burda. 1997. Reproductive characteristics and growth in the eusocial Zambian Common mole-rat (Cryptomys, sp., Bathyergidae). Zeitschrift Säugetierkunde 63: 297-306.

Bennett, N. C. and C. G. Faulkes. 2000. The African mole-rats: ecology and eusociality. Cambridge University Press. Cambridge, UK.

Bennett, N. C. and R. Navarro. 1997. Differential growth patterns between successive litters of the eusocial Damaraland mole-rat, Cryptomys damarensis, from Namibia. Journal of Zoology 241: 465473.

Blanckenhorn, W. U. 2005. Behavioral causes and consequences of sexual size dimorphism. Ethology 1016: 977-1016.

Burnham, K. and D. Anderson. 2002. Model selection and multi-model inference: a practical information-theoretic approach. Springer. Berlin, Germany.

Clutton-Brock, T. 1991. The evolution of parental care. Princeton University Press. Princeton, USA.

509 Clutton-Brock, T. H., Hodge, S. J., Spong, G., Russell, A. F., Jordan, N. R., Bennett, N. C., Sharpe,

510 L. L. et al. 2006. Intrasexual competition and sexual selection in cooperative mammals. Nature

511 444(7122): 1065-8. doi: 10.1038/nature05386.

512 Cole, T. J., Donaldson, M. D. C. and Y. Ben-Shlomo. 2010. SITAR-a useful instrument for growth curve analysis. International Journal of Epidemiology 39(6): 1558-1566. doi: 10.1093/ije/dyq115.

514 Davies, O. and K. Ku. 1977. Re-examination of the fitting of the Richards growth function.

515 Biometrics 33(3): 546-547.

516 Dengler-Crish, C. M. 2007. Phenotypic plasticity in female naked mole-rats after removal from reproductive suppression. The Journal of Experimental Biology 210: 4351-4358.

518 doi:10.1242/jeb.009399

519 Duncan, P., Portier, C., Duncan, P., Gaillard, J., Guillon, N. and A.J. Sempéré. 2000. Growth of 520 European roe deer: patterns and rates. Acta Theriologica 45: 87-94. doi: 10.4098/AT.arch.00-8.

521 English, S., Bateman, A. W. and T. H. Clutton-Brock. 2012. Lifetime growth in wild meerkats: incorporating life history and environmental factors into a standard growth model. Oecologia 169: 143-153. doi: 10.1007/s00442-011-2192-9.

524 France, J., Dijkstra, J. and M. Dhanoa. 1996. Growth functions and their application in animal 525 science. Annals de Zootechnie 45 (Supp11): 165-174. growth form and precocity at birth in eutherian mammals. Proceedings of the Royal Society B: Biological Sciences 264(1383): 859-68. doi: 10.1098/rspb.1997.0120.

529 García-Muñiz, J., Ramírez-Valverde, R., Núñez-Domínguez, and J. Hidalgo-Moreno. 2019. Dataset 530 on growth curves of boer goats fitted by ten non-linear functions. Data in Brief 23: 103672.

531 Hart, L., Chimimba, C., Jarvis, J., O'Riain, J. and N. Bennett. 2007. Craniometric sexual dimorphism 
Hauber, M. E. and E. A. Lacey. 2005. Bateman's principle in cooperatively breeding vertebrates: the effects of non-breeding alloparents on variability in female and male reproductive success. Integrative and Comparative Biology 5: 903-914.

Jarman, P. 1983. Mating systems and sexual dimorphism in large, terrestrial, mammalian herbivores. Biological Reviews 58: 485-520.

Jarvis, J. U. M., O’Riain, M. J. and E. McDaid. 1991. Growth and factors affecting body size in naked mole-rats. Pp. 358-383 in The biology of the naked mole-Rat (Sherman, P., Jarvis, J., and R. Alexander, eds). Princeton University Press. Princeton, USA.

Kilanowski, A. L. and J. L. Koprowski. 2017. Female-biased sexual size dimorphism: ontogeny, seasonality, and fecundity of the cliff chipmunk (Tamias dorsalis). Journal of Mammalogy 98(1): 204-210. doi: 10.1093/jmammal/gyw172.

Klimentidis, Y. C., Beasley, T. M., Lin, H., Murati, G., Glass, G. E., Guyton, M., et al. 2011. Canaries in the coal mine: a cross-species analysis of the plurality of obesity epidemics. Proceedings of the Royal Society B: Biological Sciences 278: 1626-1632. doi: 10.1098/rspb.2010.1890.

Korsten, P., Clutton-Brock, T., Pilkington, J. G., Pemberton, J. M. and Kruuk, L. E. B. 2009. Sexual conflict in twins: male co-twins reduce fitness of female Soay sheep. Biology letters 5(5): 663-6. doi: 10.1098/rsbl.2009.0366.

Leberg, P. L., Lehr Brisbin Jr, I., Smith, M. H. and G. C. White. 1989. Factors affecting the analysis of growth patterns of large mammals. Journal of Mammalogy 70(2): 275-283. doi: 10.2307/1381508.

Leigh, S. R. and B. T. Shea. 1996. Ontogeny of body size variation in African apes. American Journal of Physical Anthropology 99: 43-65.

Lindenfors, P., Gittleman, J. and K. Jones. 2007. Sexual size dimorphism in mammals. Pp. 16-28 in Sex, size and gender roles; evolutionary studies of sexual size dimorphism (Fairburn, D., Blanckenhorn, W., and T. Székely, T., eds). Oxford University Press. Oxford, UK.

Matějů, J. and L. Kratochvíl. 2013. Sexual size dimorphism in ground squirrels (Rodentia: Sciuridae: Marmotini ) does not correlate with body size and sociality. Frontiers in Zoology 10: 27. doi: $10.1186 / 1742-9994-10-27$.

McNamara, K. J. 1995. Sexual dimorphism: the role of heterochrony in evolutionary change and heterochrony. Pp. 65-89 in Evolutionary change and heterochrony (K. J. McNamara, ed). John Wiley and Sons.

Meyer, K. 2005. Random regression analyses using B-splines to model growth of Australian Angus cattle. Genetics, Selection, Evolution 37: 473-500.

Morfeld, K. A., Meehan, C. L., Hogan, J. N. and J. L. Brown. 2016. Assessment of body condition in African (Loxodonta africana) and Asian (Elephas maximus) elephants in North American zoos and management practices associated with high body condition scores. PloS one 11(7): e0155146. doi: 10.1371/journal.pone.0155146.

Neuenhoff, R., Cowan, D., Whitehead, H. and C. Marshall. 2011. Prenatal data impacts common bottlenose dolphin (Tursiops truncatus) growth parameters estimated by length-at-age curves. Marine Mammal Science 27: 195-216. doi: 10.1111/j.1748-7692.2010.00394.x.

O’Mara, M., Gordon, A., Catlett, K., Terranova, C. and G. Schwartz. 2012. Growth and the development of sexual size dimorphism in lorises and galagos. American Journal of Physical Anthropology 147: 11-20.

O'Riain, M. J. and J. U. M. Jarvis. 1998. The dynamics of growth in naked mole-rats: the effects of litter order and changes in social structure. Journal of Zoology 246(1): 49-60. doi: $10.1017 /$ S0952836998009066.

Parks, J. 1982. A theory of feeding and growth of animals. Springer-Verlag. Berlin, Germany. Pedersen, J. 1980. Ultrasound evidence of sexual difference in fetal size in first trimester. British 
Medical Journal 281: 1253.

Pinto, M., Jepsen, K. J., Terranova, C. J. and R. Buffenstein. 2010. Lack of sexual dimorphism in femora of the eusocial and hypogonadic naked mole-rat: A novel animal model for the study of delayed puberty on the skeletal system. Bone 46(1): 112-120. doi: 10.1016/j.bone.2009.08.060.

R Core Team. 2018. R: A language and environment for statistical computing. Vienna, Austria: R Foundation for Statistical Computing.

Ralls, K. 1976. Mammals in which females are larger than males. The Quarterly Review of Biology 51: 245-276.

Richards, F. J. 1959. A flexible growth function for empirical use. Journal of Experimental Botany 10(39): 290-300.

Scantlebury, M., Speakman, J. R. and N. C. Bennett. 2006. The energy costs of sexual dimorphism in mole-rats are morphological not behavioural. Proceedings of the Royal Society B: Biological Sciences 273: 57-63. doi: 10.1098/rspb.2005.3280.

Scharff, A., Begall, S., Grütgen, O, and Burda, H. 1999. Reproductive characteristics and growth of Zambian giant mole-rats, Cryptomys mechowi (Rodentia : Bathyergidae). Mammalia 63(2): 217-230.

Schoener, T. W. and A. Schoener. 1978. Estimating and interpreting body-size growth in some Anolis lizards. Copeia 3: 390-405.

Schulte-Hostedde, A. I. 2007. Sexual size dimorphism in rodents. Pp. 115-128 in Rodent societies: an ecological and evolutionary perspective (Wolff, J. O. and P. W. Sherman, eds). University of Chicago Press. Chicago, USA.

Shotwell, M. E., Mcfee, W. E. and E. H. Slate. 2016. A Bayesian mixture model for missing data in marine mammal growth analysis. Environmental and Ecological Statistics 23(4): 585-603. doi: 10.1007/s10651-016-0355-x.

Sikes, R. S. 2016. Guidelines of the American Society of Mammalogists for the use of wild mammals in research and education. Journal of Mammalogy 97(3): 663-688. doi: 10.1093/jmammal/gyw078.

Sofaer, H. R., Chapman, P. L., Sillett, T. S. and C. K. Ghalambor. 2013. Advantages of nonlinear mixed models for fitting avian growth curves. Journal of Avian Biology 44: 469-478. doi: 10.1111/j.1600-048X.2013.05719.x.

Starck, J. M. and R. E. Ricklefs. 1998. Avian growth and development: evolution within the altricialprecocial spectrum. Oxford University Press. Oxford, UK.

Sugden, L. G., Driver, E. A. and C. S. Kingsley. 1981. Growth and energy consumption by captive mallards. Canadian Journal of Zoology 59: 1567-1570.

Sumbera, R., Burda, H. and W. N. Chitaukali. 2003. Reproductive biology of a solitary subterranean bathyergid rodent, the silvery mole-rat (Heliophobius argenteocinereus). Journal of Mammalogy 81(1): 278-287. doi: 10.1644/1545-1542(2003)084<0278:RBOASS>2.0.CO;2

Svagelj, W. S., Gómez Laich, A. and F. Quintana. 2019. Richard's equation and nonlinear mixed models applied to avian growth: why use them? Journal of Avian Biology 50:e01864. doi: 10.1111/jav.01864.

Swanson, E. M., McElhinny, T. L., Dworkin, I., Weldele, M. L., Glickman, S. E. and K. E. Holekamp. 2013. Ontogeny of sexual size dimorphism in the spotted hyena (Crocuta crocuta). Journal of Mammalogy 94(6): 1298-1310. doi: 10.1644/12-MAMM-A-277.1.

Teleken, J. T., Galvão, A. C. and S. Robazza, S. 2017. Comparing non-linear mathematical models to describe growth of different animals. Acta Scientarium 39: 73-81. doi: 10.4025/actascianimsci.v39i1.31366.

Thorley, J. 2019. The life history of Damaraland mole-rats Fukomys damarensis: growth, ageing and behaviour. PhD Thesis. University of Cambridge. 
Thorley, J., Katlein, N., Goddard, K., Zöttl, M. and T. Clutton-Brock. 2018. Reproduction triggers adaptive increases in body size in female mole-rats. Proceedings of the Royal Society B: Biological Sciences 285: 20180897. doi: https://doi.org/10.5061/dryad.d783p98.

Tjørve, E. and K. M. C. Tjørve. 2010. A unified approach to the Richards-model family for use in growth analyses : Why we need only two model forms. Journal of Theoretical Biology 267(3): 417425. doi: $10.1016 /$ j.jtbi.2010.09.008.

Tjørve, K. M. C. and E. Tjørve. 2017a. A proposed family of Unified models for sigmoidal growth. Ecological Modelling 359: 117-127. doi: 10.1016/j.ecolmodel.2017.05.008.

Tjørve, K. and E. Tjørve, E. 2017b. Modelling avian growth with the Unified-Richards: as exemplified by wader-chick growth. Journal of Avian Biology 48: 770-784. doi: 10.1111/jav.00992.

Tonner, P. D., Darnell, C. L., Engelhardt, B. E. and A. K. Schmid. 2017. Detecting differential growth of microbial populations with Gaussian process regression. Genome Research 27: 320-333. doi: 10.1101/gr.210286.116.Freely.

Vrána, J., Remeš, V., Matysioková, B., Tjørve, K. M. C. and E. Tjørve. 2019. Choosing the right sigmoid growth function using the unified-models approach. Ibis 161: 13-26. doi: 10.1111/ibi.12592.

White, I. M. S., Thompson, R. and S. Brotherstone. 1999. Genetic and environmental smoothing of lactation curves with cubic splines. Journal of Dairy Science 82: 632-638.

Wilson, D. E. and R. Mittermeier (eds). 2014. Handbook of the mammals of the world, 4: sea mammals. Lynx Ediciones. Barcelona, Spain.

Young, A. and N. C. Bennett. 2013. Intra-sexual selection in cooperative mammals and birds: why are females not bigger and better armed? Philosophical transactions of the Royal Society of London. Series B, Biological Sciences 368: 20130075. doi: 10.1098/rstb.2013.0075.

Young, A. J. and N. C. Bennett. 2010. Morphological divergence of breeders and helpers in wild Damaraland mole-rat societies. Evolution 64(11): 3190-3197. doi: 10.1111/j.15585646.2010.01066.x.

Zach, R., Rigby, L. and K. R. Mayoh. 1984. Growth curve analysis of birds: the Richards model and procedural problems. Canadian Journal of Zoology 62: 2429-2435.

Zöttl, M., Thorley, J., Gaynor, D., Bennett, N. C. and T. Clutton-Brock. 2016. Variation in growth of Damaraland mole-rats is explained by competition rather than by functional specialization for different tasks', Biology Letters 12, p. 20160820. doi: 10.1098/rsbl.2016.0820.

Zullinger, E. M., Ricklefs, R. E., Redford, K. H. and G. M. Mace. 1984. Fitting sigmoidal equations to mammalian growth curves. Journal of Mammalogy 65(4). 607-636. doi: 10.2307/1380844.

\section{FIGURE LEGENDS}

Fig. 1: The cumulative use of sigmoid-like functions to model size-at-age data in the mammalian literature since 1980. Black fill refers to studies that have employed a Richards function (irrespective of whether other functions were also fitted alongside the Richards). Grey fill refers to studies that employed other two- or three-parameter growth functions (including Brody, logistic, monomolecular, Gompertz, von Bertalanffy, quadratic); when hatched, multiple comparisons were made between these 
different functions, and when solid, the study only tested a single growth function. See the main text Supplementary Data S1 for details of literature searching.

Fig. 2: Sex-specific patterns of skeletal growth in captive Damaraland mole-rats: A) incisor width, B) body length. Left hand plots display the predicted growth curves from the best fitting unified growth model for each trait (females- solid line, males- dashed line), with the points showing the raw data (females- crossed, males- circles). Right hand plots show the instantaneous growth rate (growth velocity) of each trait, here taken as the first derivative (gradient) of general additive mixed models fitted to morphological datasets with a smoother for age (females- solid, males- dashed).

Fig. 3: Skull growth (incisor width) of wild Damaraland mole-rats. As individuals are of unknown age, asymptotic mass and growth rate were estimated from a von Bertalanffy growth curve reparametrized as an interval equation (Schoener \& Schoener 1978). The equation models the change in size across repeated capture events as a function of the time difference between capture events and initial size. Figures A) and B) display the raw data for repeated captures on females and males, respectively; each slope displays the change in incisor width across a single recapture event: i.e. from $t_{1}$ to $t_{2}$. Note that with increasing initial incisor width, the slope of the change in incisor width converges on the estimated population-level asymptote, highlighted by the horizontal black line. C) Fitted growth rates for females (solid line) and males (dashed line) with changing initial incisor width; each point represents a single slope from A and B.

Fig. 4: Body length growth of wild Damaraland mole-rats. A) and B) display the raw data for repeated captures on females and males, respectively, with the thicker black line showing the population-level asymptote estimate from the best-fitting models. C) Fitted body length growth rates for females (solid line) and males (dashed line) with changing initial body length width; each point represents a single slope from A and B. 
694 Fig. 5: Male:female body length dimorphism in Rodentia. Data provided for 110 species across 8 695 Rodent families (Bathyergidae (2), Chinchillidae (1), Ctenomyidae (1), Geomyidae (4), Heteromyidae 696 (50), Muridae (29), Sciuridae (22), Zapodidae (2)), taken from Schulte-Hostedde (2007). Mean across 697 rodents $=1.04$ (dashed line; $1 \mathrm{SD}=0.004)$. Value for wild Damaraland mole-rats, as predicted by 698 models, shown by the solid vertical line: 1.04 .

699

700 Supplementary Data S3. Instantaneous growth rates (growth velocity) for the unified growth 701 models applied to female incisor width (a), male incisor width (b), female body length (c), 702 and male body length (d). Instantaneous growth rates represent the first derivative of the best703 fitting non-linear mixed effects model in each case. Points on each curve highlight the estimated point of maximum growth in either model. All models were fitted to data on known-age, captive individuals. 
Table 1. Parameterization of unified models of sigmoid growth. $S_{t}$ is the size at age $\mathrm{t}$ days; $A, k, T_{i}$ and the upper asymptote, the maximum relative growth rate, and the timing of inflection point, respectively; $d$ shifts the inflection value vertically. $W_{i}$ is the absolute value at maximum growth (i.e. size at inflection point), which can also be expressed as a percentage of the upper asymptote.

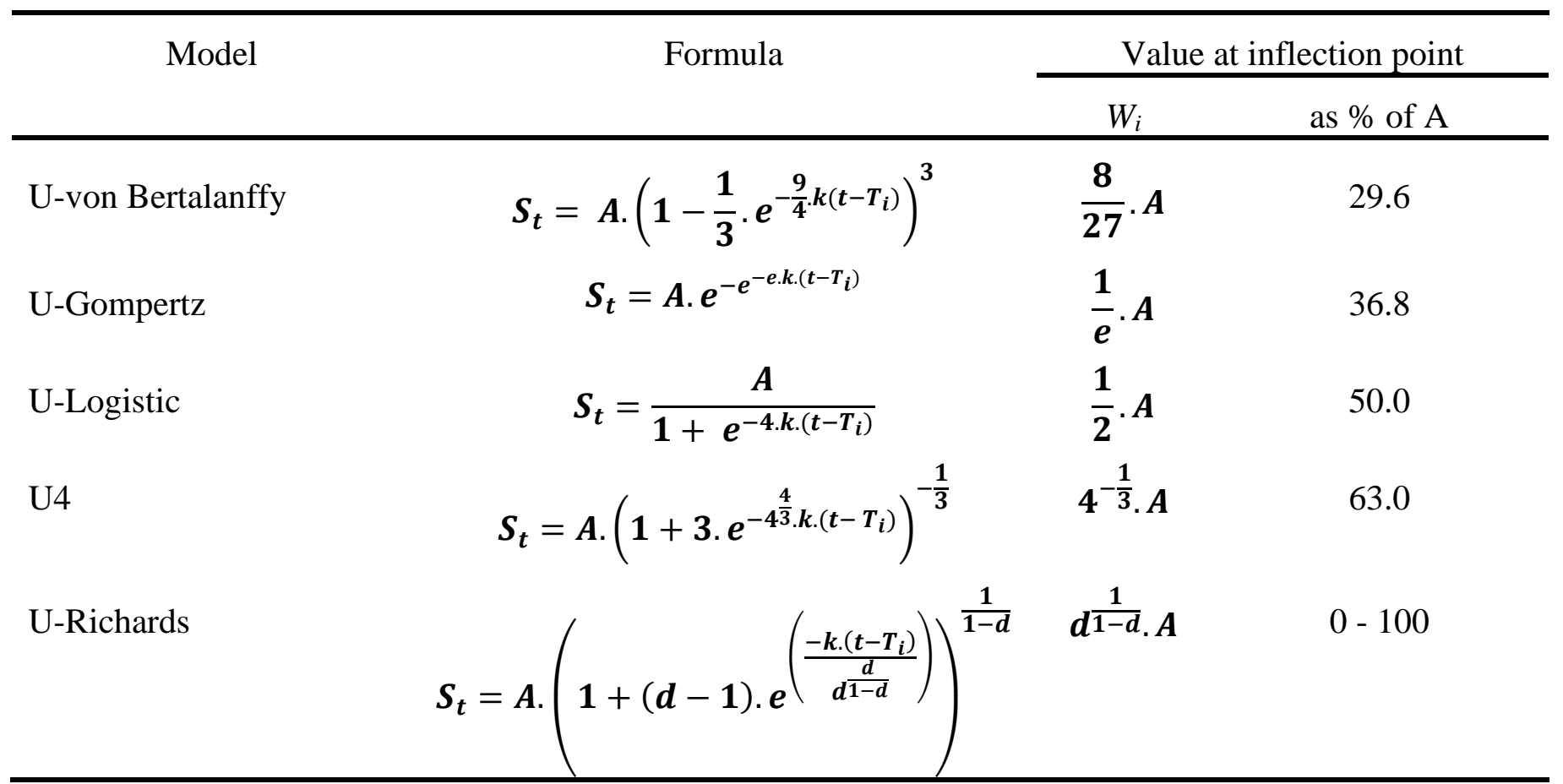


Table 2. Model comparisons of skeletal growth in Damaraland mole-rats. Female incisor width $d=0.82$, Male incisor width $d=1.45$, female body length $d=0.50$, male body length $d=2.87$.

\begin{tabular}{lcccccccc}
\hline \multicolumn{1}{c}{ Model } & $\mathrm{k}$ & AIC & $\Delta$ AIC & Weights & $\mathrm{k}$ & AIC & $\Delta$ AIC & Weights \\
\hline Incisor Width & & & & & & & & \\
U-von Bertalanffy & 8 & -89.18 & 0.00 & 0.65 & 9 & 267.69 & 29.37 & 0.00 \\
U-Gompertz & 8 & -86.27 & 2.90 & 0.16 & 9 & 246.1 & 7.78 & 0.02 \\
U-Logistic & 9 & -65.23 & 23.94 & 0.00 & 9 & 247.23 & 8.91 & 0.01 \\
U4 & 9 & 32.48 & 121.66 & 0.00 & 9 & 377.29 & 138.97 & 0.00 \\
U-Richards & 9 & -86.56 & 2.62 & 0.18 & 10 & 238.32 & 0.00 & 0.97 \\
& & & & & & & & \\
Body Length & & & & & & & & \\
U-von Bertalanffy & 8 & 2490.11 & 5.64 & 0.06 & 8 & 2697.85 & 29.58 & 0.00 \\
U-Gompertz & 8 & 2494.63 & 10.16 & 0.00 & 8 & 2689.65 & 21.37 & 0.00 \\
U-Logistic & 9 & 2507.92 & 23.45 & 0.00 & 8 & 2671.17 & 2.89 & 0.15 \\
U4 & 9 & 2533.27 & 48.80 & 0.00 & 8 & 2670.24 & 1.97 & 0.23 \\
U-Richards & 9 & 2484.47 & 0.00 & 0.94 & 9 & 2668.28 & 0.00 & 0.62 \\
\hline
\end{tabular}


Table 3. Growth parameter estimates from models fitted to skeletal traits on captive Damaraland mole-rats. A is the upper asymptote, $k$ the relative maximum growth rate, $T_{i}$ the time of inflection, and $d$ the shape parameter that controls the inflection value. The size at birth, $\mathrm{W}_{0}$, and the absolute maximum growth rate, $\mathrm{g}_{\max }$, are estimated from model outputs. SSD is calculated from equivalent models. Random effects variation in Table SD2.

\begin{tabular}{|c|c|c|c|c|c|c|c|c|c|}
\hline & & $A(\mathrm{SE})$ & $k(\mathrm{SE})$ & $T_{i}(\mathrm{SE})$ & $W_{0}$ & $d$ & $\begin{array}{c}\text { Time to reach } 90 \% \\
\text { Asymptotic Mass, years }\end{array}$ & $\begin{array}{c}\mathrm{g}_{\max }, \\
\mathrm{mm} / \mathrm{day}\end{array}$ & $\begin{array}{l}\mathrm{m}: \mathrm{f} \\
\text { SSD }\end{array}$ \\
\hline \multicolumn{10}{|l|}{ Females } \\
\hline \multirow[t]{5}{*}{ Incisor Width } & U-von Bertalanffy & $6.59(0.04)$ & $0.00194(0.00004)$ & $3.65(2.85)$ & 1.91 & - & 1.44 & 0.013 & - \\
\hline & U-Gompertz & $6.57(0.02)$ & $0.00172(0.00004)$ & $32.04(2.51)$ & 2.06 & - & 1.41 & 0.011 & - \\
\hline & U-Logistic & $6.48(0.03)$ & $0.00147(0.00003)$ & $101.79(2.34)$ & 2.30 & - & 1.31 & 0.010 & - \\
\hline & $\mathrm{U} 4$ & $6.45(0.03)$ & $0.00121(0.00003)$ & $189.08(2.56)$ & 2.69 & - & 1.28 & 0.008 & - \\
\hline & U-Richards & $6.58(0.04)$ & $0.00183(0.00003)$ & $17.47(2.67)$ & 2.17 & 0.82 & 1.42 & 0.012 & - \\
\hline \multicolumn{10}{|l|}{ Males } \\
\hline \multirow[t]{5}{*}{ Incisor Width } & U-von Bertalanffy & $7.65(0.04)$ & $0.00180(0.00004)$ & $39.07(2.15)$ & 1.73 & - & 1.64 & 0.014 & 1.16 \\
\hline & U-Gompertz & $7.58(0.04)$ & $0.00167(0.00003)$ & $74.00(2.00)$ & 1.86 & - & 1.57 & 0.013 & 1.15 \\
\hline & U-Logistic & $7.42(0.04)$ & $0.00146(0.00003)$ & $147.59(2.09)$ & 2.20 & - & 1.44 & 0.011 & 1.15 \\
\hline & $\mathrm{U} 4$ & $7.26(0.04)$ & $0.00132(0.00002)$ & $232.22(2.50)$ & 2.59 & - & 1.32 & 0.012 & 1.13 \\
\hline & U-Richards & $7.50(0.04)$ & $0.00154(0.00003)$ & 111.60 (1.99) & 3.39 & 1.45 & 1.50 & 0.012 & 1.14 \\
\hline \multicolumn{10}{|l|}{ Females } \\
\hline \multirow[t]{5}{*}{ Body Length } & U-von Bertalanffy & $18.49(0.08)$ & $0.00194(0.00005)$ & $-119.13(6.40)$ & 9.53 & - & 1.10 & 0.036 & - \\
\hline & U-Gompertz & $18.46(0.08)$ & $0.00167(0.00004)$ & $-97.88(5.90)$ & 9.72 & - & 1.63 & 0.031 & - \\
\hline & U-Logistic & $18.36(0.08)$ & $0.00130(0.00003)$ & $-38.5(4.71)$ & 10.09 & - & 1.27 & 0.029 & - \\
\hline & $\mathrm{U} 4$ & $18.42(0.07)$ & $0.00097(0.00003)$ & $46.08(3.75)$ & 10.76 & - & 1.06 & 0.018 & - \\
\hline & U-Richards & $18.56(0.08)$ & $0.00399(0.00010)$ & $-167.11(7.66)$ & 9.02 & 0.00 & 1.13 & 0.074 & - \\
\hline
\end{tabular}


Males

\begin{tabular}{|c|c|c|c|c|c|c|c|c|c|}
\hline \multirow[t]{5}{*}{ Body Length } & U-von Bertalanffy & $19.82(0.08)$ & $0.00204(0.00005)$ & $-77.36(5.01)$ & 8.92 & - & 1.15 & 0.040 & 1.07 \\
\hline & U-Gompertz & $19.79(0.08)$ & $0.00177(0.00004)$ & $-55.03(4.55)$ & 9.19 & - & 1.13 & 0.035 & 1.07 \\
\hline & U-Logistic & $19.71(0.08)$ & $0.00137(0.00003)$ & $1.66(3.58)$ & 9.82 & - & 1.10 & 0.027 & 1.07 \\
\hline & U4 & $19.61(0.07)$ & $0.00109(0.00003)$ & $85.93(2.83)$ & 10.53 & - & 1.07 & 0.021 & 1.06 \\
\hline & U-Richards & $19.68(0.07)$ & $0.00120(0.00003)$ & $41.32(3.08)$ & 11.22 & 2.87 & 1.09 & 0.024 & 1.06 \\
\hline
\end{tabular}




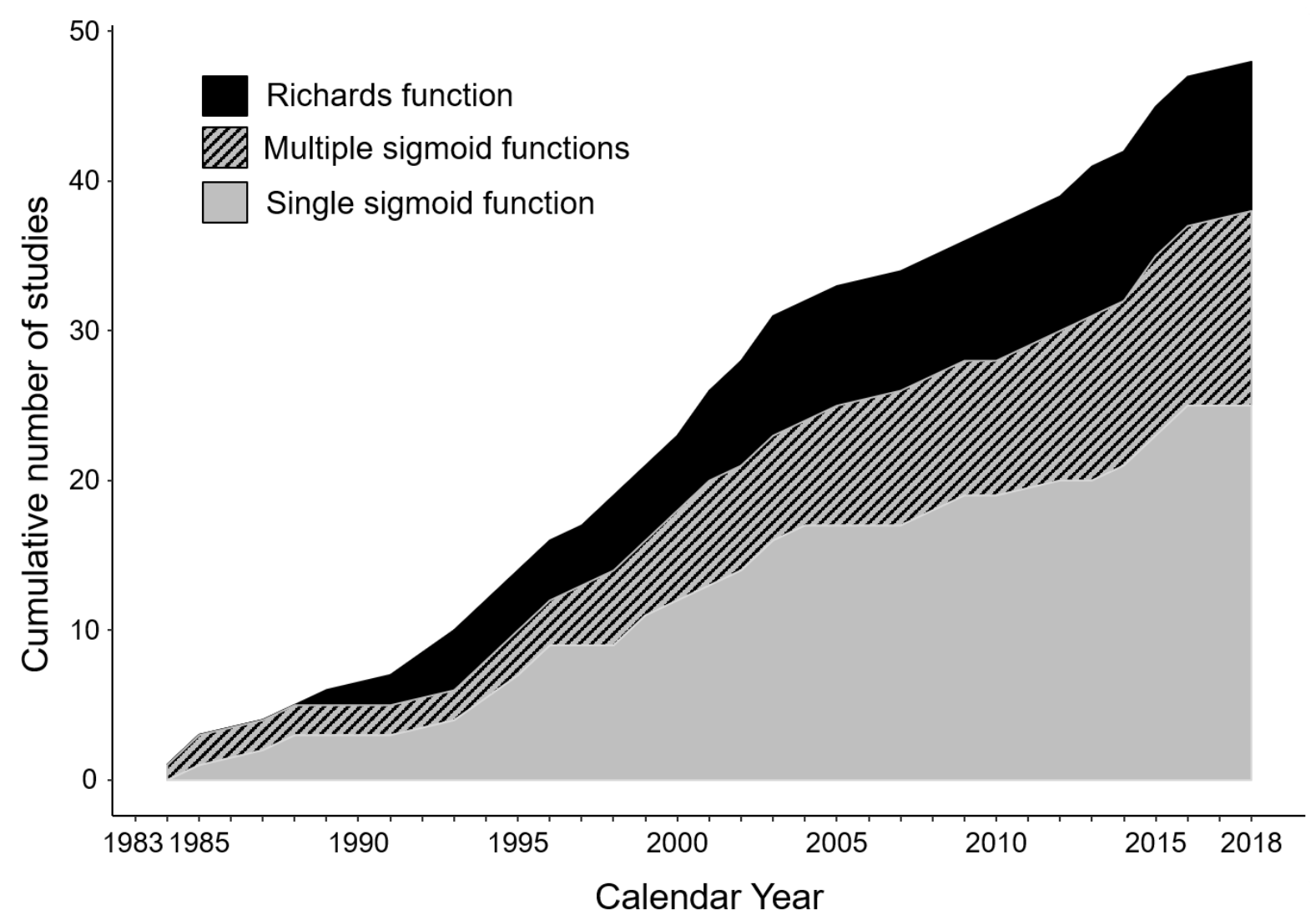

Fig. 1 
A
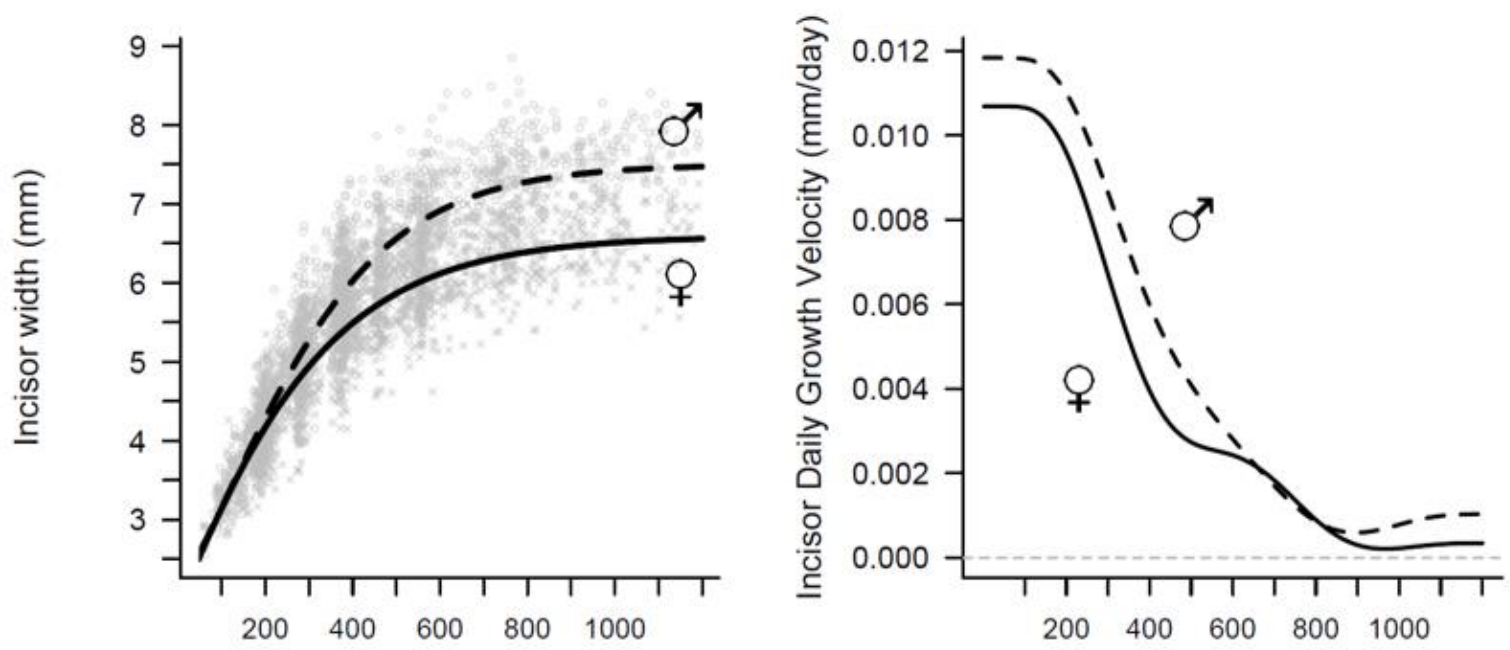

B
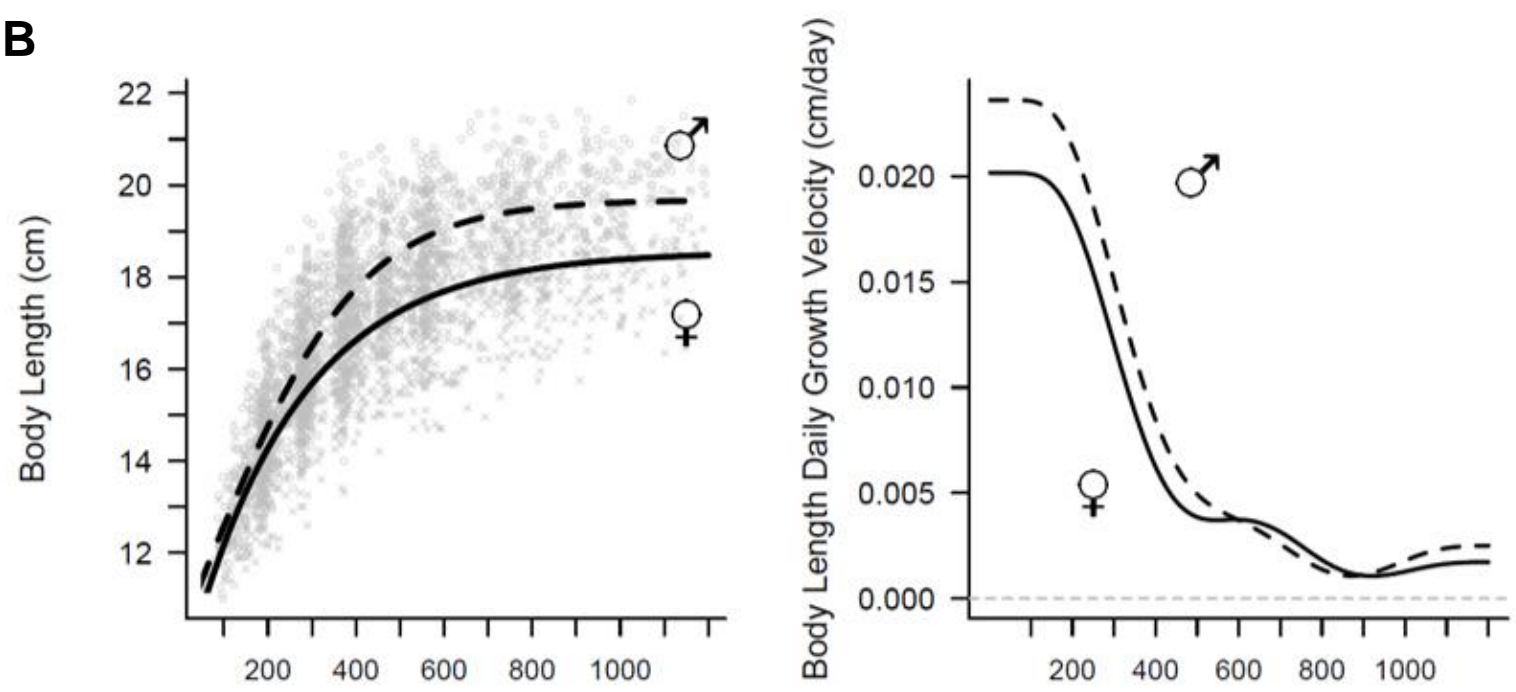

Age (days)

Age (days)

Fig. 2

768

769

770

771

772 


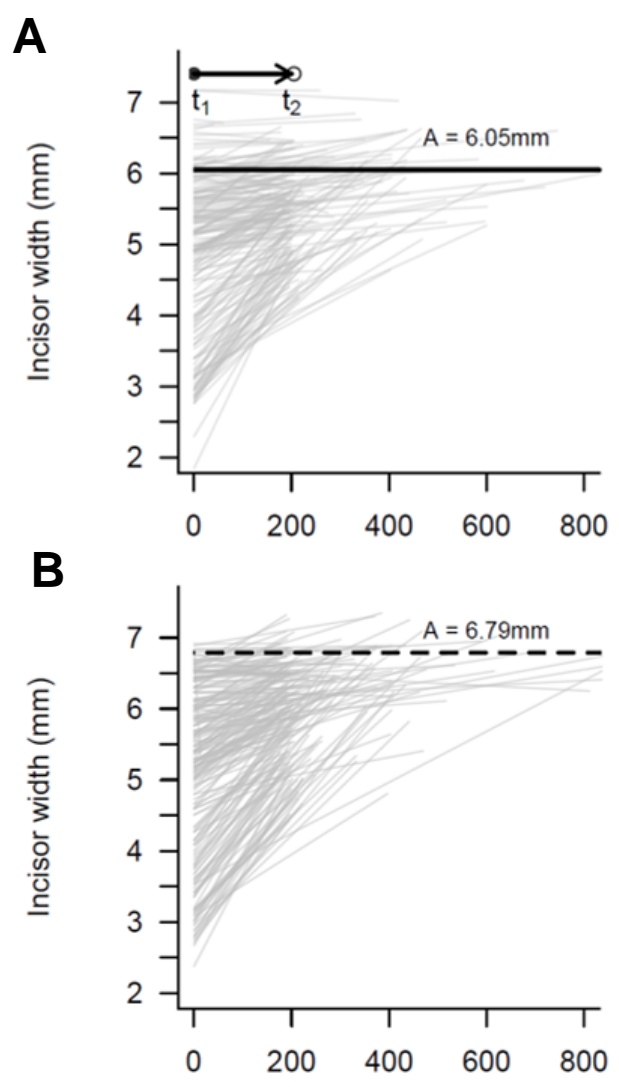

Time between successive measures per individual (days)

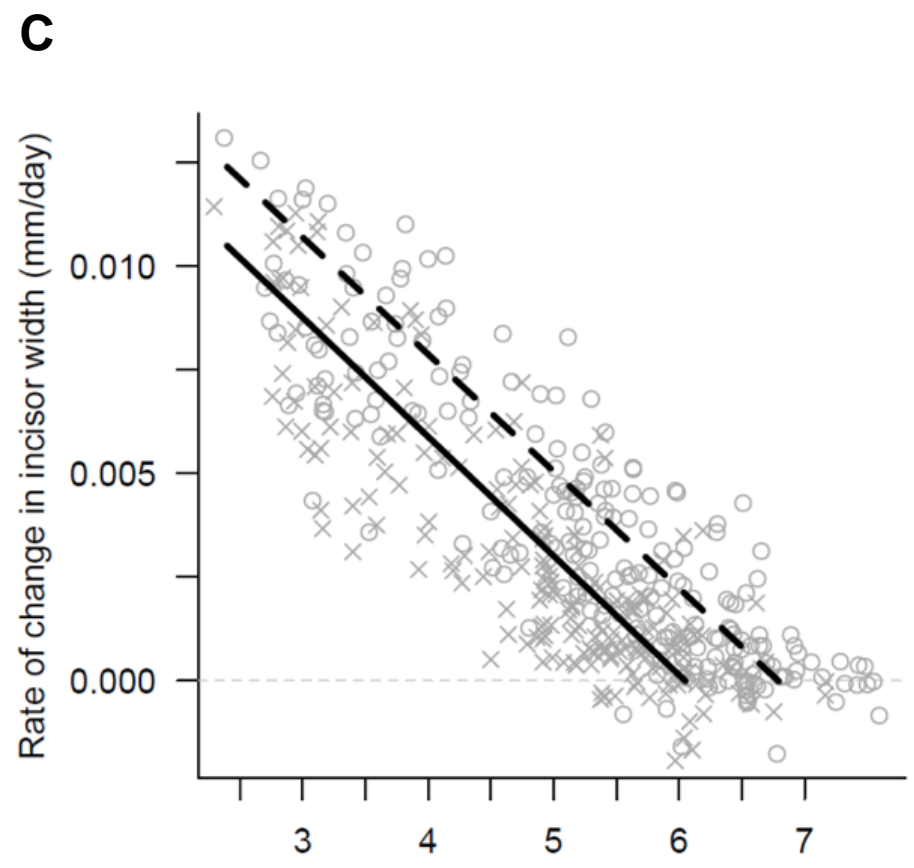

Incisor width (mm)

Fig. 3 
A

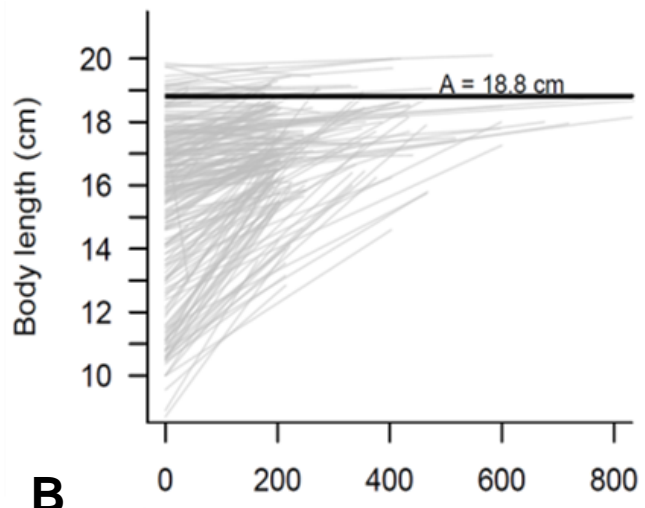

C

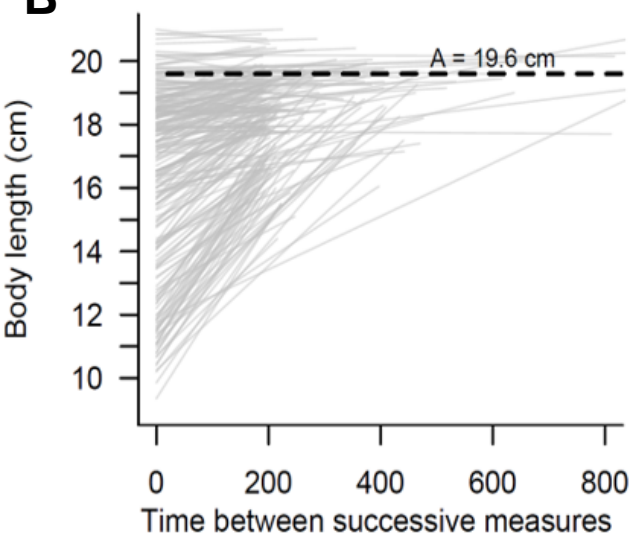

Time between successive measures per individual (days)

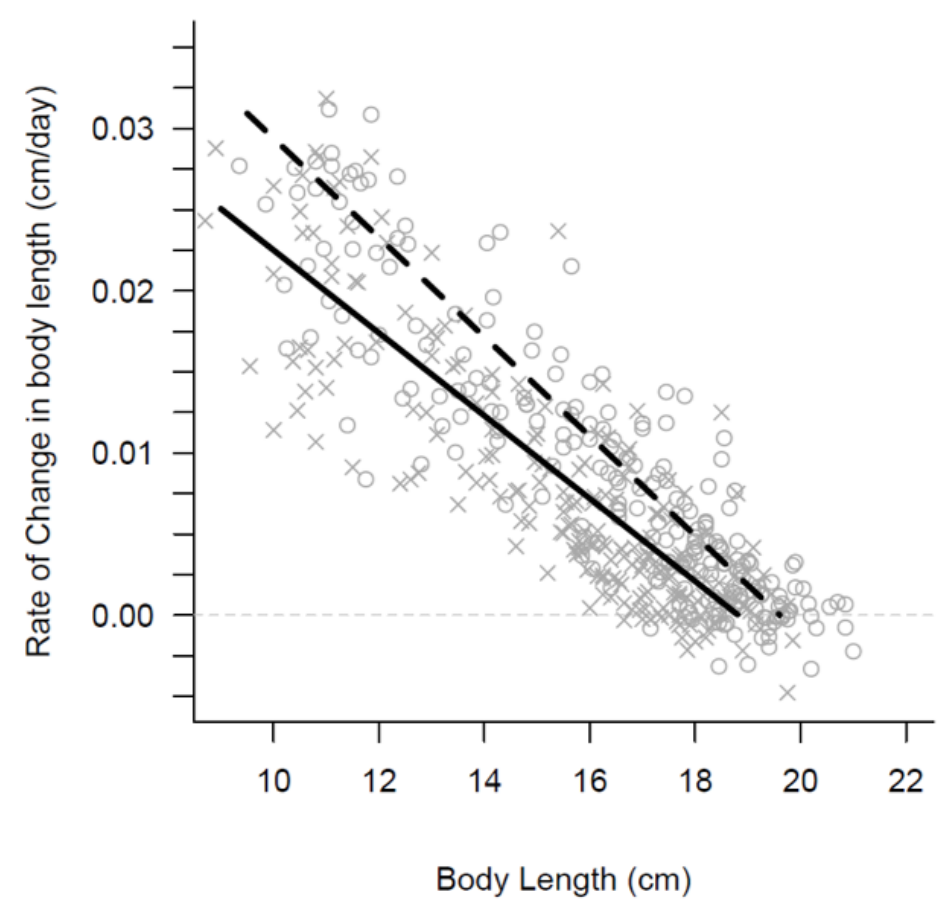

795

796

797

798

799

800

801

802

803

804

805

806

807

808

809

810

811

812

Fig. 4 


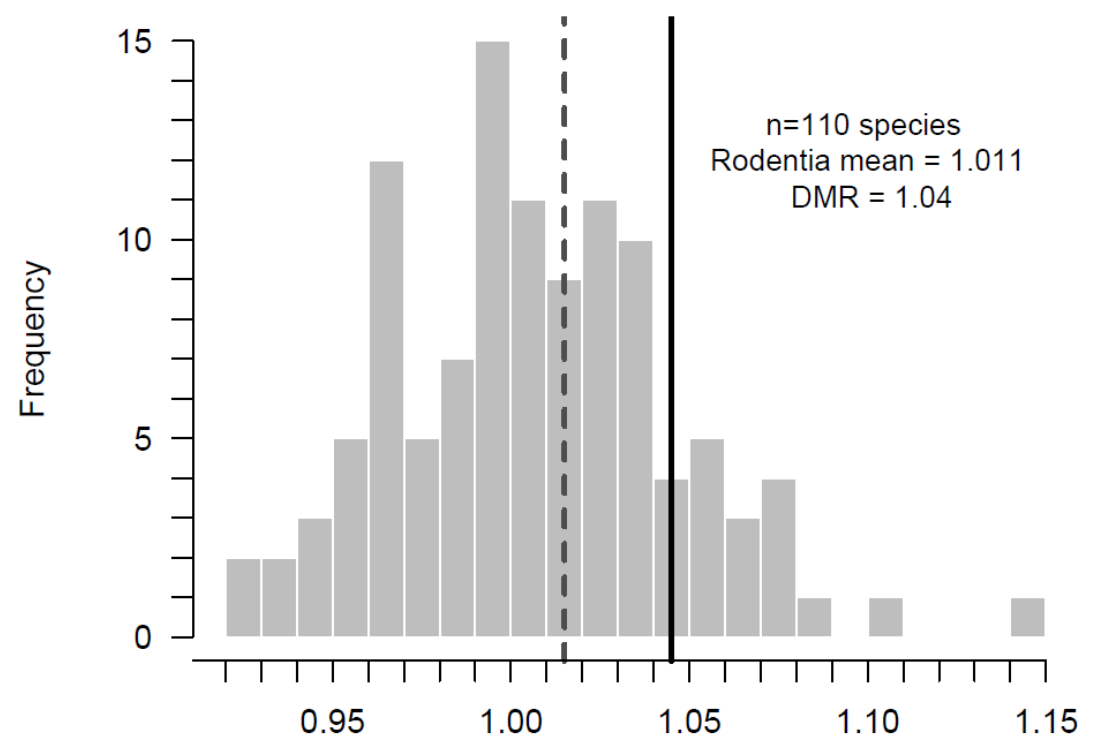

Male:female body length dimorphism

Fig. 5

816

817

818

819

820

821

822

823

824

825

826

827

828

829

830

831

832

833

834

835

836

837

838

839

840

841

842

843

844

845

846 